\title{
Percurso do idoso em redes de atenção à saúde: um elo a ser construído
}

I 1 Carla Targino Bruno dos Santos, ${ }^{2}$ Luiz Odorico Monteiro de Andrade,

${ }^{3}$ Maria Josefina Silva, ${ }^{4}$ Maria Fátima de Sousa I

Resumo: Objetivamos compreender a continuidade do cuidado prestado ao idoso nas redes de atenção à saúde de Fortaleza. É um estudo qualitativo descritivo, utilizamos grupos focais, entrevistas semiestruturadas, e discurso do sujeito coletivo para obtenção e organização dos dados. Na Rede de Atenção Saúde da Família, observamos deficit em infraestrutura e profissionais. $\mathrm{Na}$ Especializada, constatamos uma demanda reprimida diante da carência em serviços especializados. $\mathrm{Na}$ Hospitalar, o fluxo de informações é deficiente. $\mathrm{Na}$ Urgência e Emergência, o serviço móvel prioriza a remoção e no hospital, o atendimento conforme a gravidade. A estreita relação entre as Redes de Saúde Mental e Saúde da Família, contribui para a continuidade efetiva do cuidado. Concluímos que a atenção à saúde do idoso no sistema é frágil e descontinuada, comprometendo a consolidação da integralidade.

> Palavras-chave: serviços de saúde para idosos; assistência à saúde; idoso.

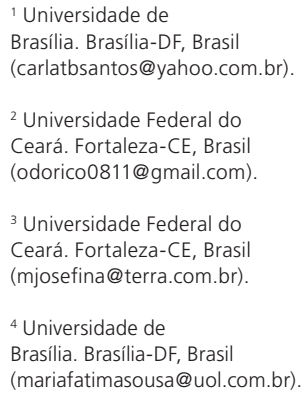

Recebido em: 08/12/2014 Aprovado em: 29/06/2015 
A partir da segunda metade do século XX, a queda da natalidade, mais acentuada que a taxa de mortalidade, resultou na redução no crescimento populacional brasileiro. Concomitantemente, observamos aumento na expectativa de vida ao nascer, que passa de 62,57 anos em 1980 para 73,17 anos em 2009, e poderá chegar a 81,29 anos em 2050, gerando aumento significativo na população de idosos. Pensando na população mundial, a esperança de vida ao nascer em 2009 era em média de 68 anos, sendo San Marino e Japão os países com maior longevidade (83 anos) e Malawi com a menor longevidade (47 anos). Esse quadro resulta numa intensa alteração na estrutura populacional e padrões de morbimortalidade, gerando novas demandas sociais, especialmente para o setor da saúde (IBGE, 2012; WHO, 2012).

O envelhecimento populacional é reconhecido como uma das principais conquistas sociais do século XX, trazendo grandes desafios para as políticas públicas do mundo. Um dos maiores desafios é assegurar que o desenvolvimento econômico e social ocorra de forma contínua, garantindo um patamar econômico mínimo para a manutenção da dignidade humana, equidade, direitos e responsabilidades sociais entre grupos etários. Nos países desenvolvidos, este fenômeno aconteceu ao longo do século XX, num momento socioeconômico favorável, o que permitiu a expansão de seus sistemas de proteção social. Nos países em desenvolvimento e, especificamente, no caso brasileiro, o acelerado envelhecimento está ocorrendo em meio a uma conjuntura recessiva e a uma crise fiscal que dificultam a expansão do sistema de proteção social para todos os grupos etários e, em particular, para os idosos (CAMARANO; PASINATO, 2004).

O Brasil estruturou-se em relação ao envelhecimento populacional por meio de políticas nacionais voltadas para esta população. Isso aconteceu primeiramente em 1994, com a Política Nacional do Idoso, a qual objetiva sobretudo assegurar os direitos sociais do idoso, criando condições para promover sua autonomia, integração e participação efetiva na sociedade. Em seguida foi publicado o Estatuto do Idoso, em 2003, que trata das prioridades, garantias e direitos desses usuários. Posteriormente foram criados a Política Nacional de Saúde da Pessoa Idosa e o Pacto de Saúde, em 2006, que traz a saúde desta população entre suas seis prioridades (BRASIL, 1994, 2003, 2006a, 2006b). 
A American Geriatrics Society recomendou uma reavaliação dos papéis e

implantação de serviços com atuação em geriatria. Os sistemas de saúde usam uma variedade de estratégias para potencializar suas experiências geriátricas, tendendo a concentrar recursos desta medicina sobre os idosos, definida como a mais necessitada. O programa de medicina geriátrica inclui locais de tratamento em regime de internamento, ambulatório, casa de repouso e domiciliário. Essa prática clínica complementa o atendimento prestado aos idosos na atenção primária, e os médicos da atenção primária tendem a encaminhar para especialistas em medicina geriátrica os idosos com deficiência avançada ou síndromes geriátricas (CHRISTOPHER et al., 2008, p. 1802).

O município de Fortaleza-Ceará, quinta cidade mais populosa do país, possui um sistema administrativo descentralizado, dividido em seis secretarias executivas regionais, cada uma dispondo de um distrito de saúde, de educação, meio ambiente, finanças, assistência social e infraestrutura. A Gestão Municipal de Saúde conceitua a saúde como um objeto complexo, produzido socialmente, e cuja elaboração requer uma abordagem transdisciplinar, intersetorial e multiprofissional, possibilitando a consolidação do modelo de cuidado integral à saúde estruturado por meio dos arranjos organizacionais de cinco redes de atenção à saúde (RA): RA da Estratégia Saúde da Família (RA ESF), RA da Atenção Especializada ou Ambulatorial (RA ESP), RA da Urgência e Emergência (RA UE), RA Hospitalar (RA HOSP) e RA da Saúde Mental (RA SM), bem como de três redes de serviços: Vigilância Epidemiológica, Vigilância Sanitária e Vigilância Ambiental (FORTALEZA, 2007; ANDRADE, 2008).

Consideramos o conceito de Redes de Atenção à Saúde como um conjunto de açōes e serviços articulados, em níveis de complexidade crescentes, com a finalidade de garantir a integralidade da atenção, transversalizando todo o sistema, desenhando o que entendemos por linha de cuidado, que por sua vez, entendemos como o conjunto de saberes, tecnologias e recursos necessários ao enfrentamento de determinados riscos, agravos ou condiçōes específicas do ciclo de vida, a serem ofertados de forma oportuna, articulada e contínua pelo sistema de saúde, entendendo sua implementação como estratégia central da organização e qualificação das redes, com vistas à integralidade do cuidado (ANDRADE, 2008; BRASIL, 2011; BRAGA, 2006). 
A figura 1 representa o caminho que o usuário percorre nas redes de atenção à saúde, de forma que receba um cuidado contínuo e integral (ANDRADE, 2006, p.783).

Figura 1. Espiral da complexidade da linha de cuidado e matriciamento nas redes de atenção

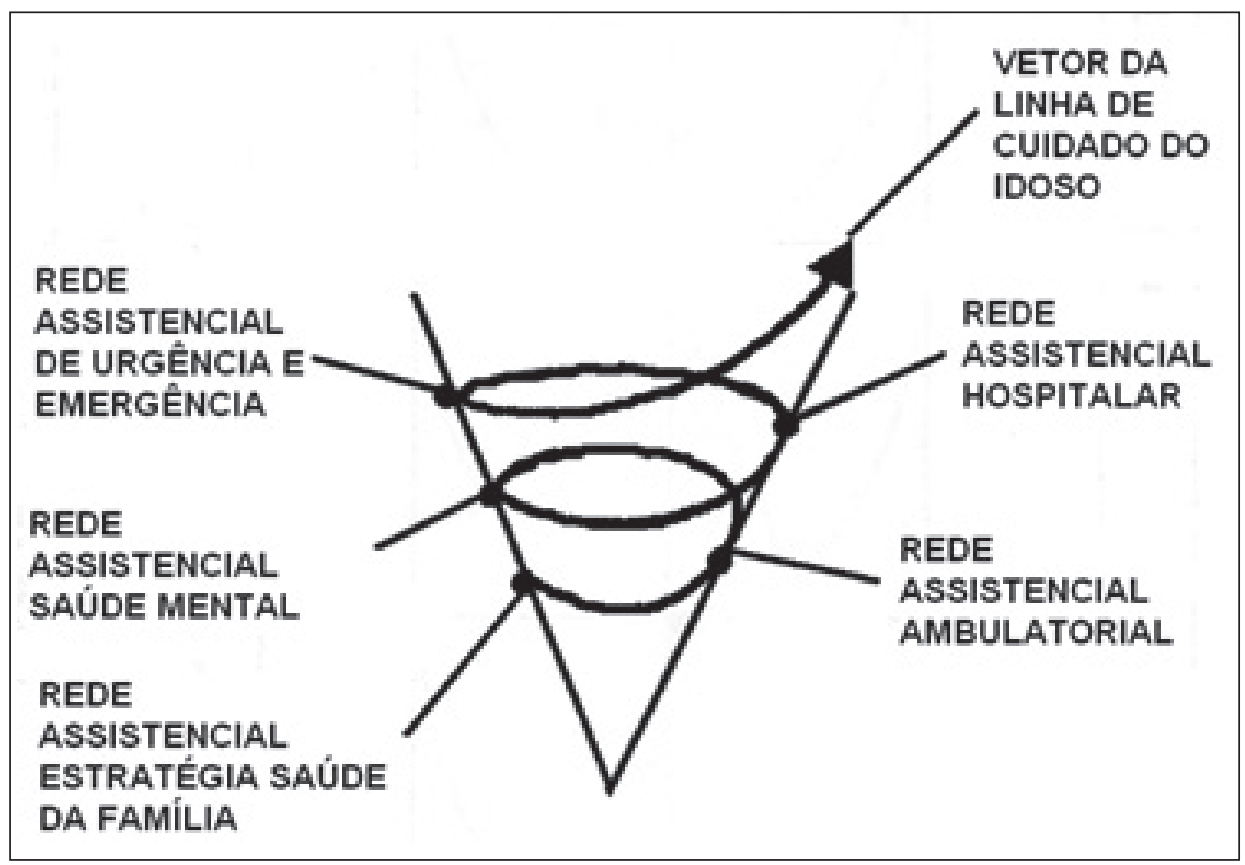

Fonte: Andrade et al. (2006).

Observamos duas concepções de linha de cuidado, uma com dimensão mais estática, que descreve seus princípios, características e finalidades, e outra dimensão, dinâmica, a qual delineia o percurso que o usuário faz ao receber os cuidados, de forma linear e contínua nos serviços de saúde, sem contraposições, mas complementaridades. Compreendemos, para efeito deste estudo, linha de cuidado como o cuidado, com seu saber científico e tecnológico, destinado ao indivíduo, direcionado ao momento em que ele se encontra no ciclo de vida, possuindo um agravo à saúde ou não, de forma contínua e articulada entre as diferentes redes de atenção à saúde e rede de serviços em seus respectivos níveis de atenção.

Ao conhecermos o percurso do idoso em rede e clarificarmos o processo de cuidado destinado a ele, com suas peculiaridades e desnivelamentos, 
auxiliaremos nas tomadas de decisão na rotina diária dos serviços de saúde, personificando esta assistência, reduzindo os fatores associados às morbidades, ou seja, instrumentalizaremos os serviços para o desenvolvimento de um cuidado integral a esta população. Permitiremos ainda a observação do desempenho das redes de atenção ao dispensar esse cuidado, obtendo informações sobre o funcionamento dos serviços, bem como seus pontos positivos, deficitários, e o que ainda está em elaboração.

A análise da linha de cuidado possibilitará o mapeamento dos recursos disponíveis nos diversos segmentos da saúde, avaliando as tecnologias utilizadas para o atendimento do usuário, como: tipo de assistência, fluxos, mecanismos de regulação, bloqueios no acesso aos serviços, ações de vigilância à saúde, promoção e utilização dos recursos especializados (MALTA; MERHY, 2010, p. 593).

Este estudo tem como objetivo compreender, por meio da percepção dos gestores, como ocorre a continuidade do cuidado do idoso nas cinco redes de atenção à saúde de Fortaleza. ${ }^{1}$

\section{Caminhos percorridos}

Trata-se de pesquisa de abordagem qualitativa realizada no município de Fortaleza, Ceará, Brasil. Utilizamos a técnica de grupos focais (GF) e entrevistas semiestruturadas, uma vez que não foi possível reunir os participantes das RA HOSP, RA ESP e da RA UE.

Os informantes implicados ocupam cargo na gestão ou coordenação em alguma das cinco redes de atenção à saúde. Tivemos um número total de 18 participantes. Seis coordenadores das Secretarias Executivas Regionais (SERs) compondo o GF da RA ESF, cinco coordenadores dos Centros de Atenção Picocossocial (CAPS) no GF da RA SM. Na RA ESP participaram três gestores, e na RA de UE e na RA HOSP, dois gestores cada. Pretendemos compreender como os profissionais de saúde, que coordenam e vivenciam o cuidado dispensado ao idoso, percebem e operacionalizam a linha de cuidado desta população no seu ambiente de trabalho.

As perguntas apresentadas como norteadoras das interaçôes nos GF e entrevistas semiestruturadas foram: qual o cuidado destinado ao idoso na sua unidade de saúde de atuação? Como acontecem o encaminhamento e o retorno de informações sobre o idoso na sua unidade de saúde? 
Registramos as entrevistas e GF em aparelhos de MP3, gravadores e por meio de anotações realizadas pelas pesquisadoras, moderadora e observadora. Alguns participantes escreveram sobre o que foi discutido e acerca das perguntas norteadoras, enviaram por correio eletrônico e por escrito essas reflexões (neste caso, entregues em mãos para a pesquisadora), e fizeram parte dos resultados do estudo. As falas gravadas foram transcritas por meio de escuta repetitiva e digitação em computador.

Identificamos os participantes conforme o quadro 1, a seguir (Santos, 2009).

Quadrro 1. Identificação dos participantes da pesquisa

\begin{tabular}{|l|l|}
\hline \multicolumn{1}{|c|}{ GRUPOS FOCAIS } & \multicolumn{1}{c|}{ SIGLAS DE IDENTIFICAÇÃO } \\
\hline GF RA Estratégia Saúde da Família & ESF1, ESF2, ESF3, ESF4, ESF5 e ESF6 \\
\hline GF RA Especializada ou Ambulatorial & ESP1, ESP2 e ESP3 \\
\hline GF RA Hospitalar & HOSP1 e HOSP2 \\
\hline GF RA Urgência e Emergência & UE1 e UE2 \\
\hline GF RA Saúde Mental & SM1, SM2, SM3, SM4 e SM5 \\
\hline
\end{tabular}

Fonte: Santos (2009).

As informações apreendidas nos GF e entrevistas foram organizadas utilizando o Discurso do Sujeito Coletivo (DSC), proposta de organização e tabulação de dados qualitativos de natureza verbal, obtidos de depoimentos, artigos de jornal, matérias de revistas, cartas, dentre outros (Lefevre, Lefevre, 2003).

\section{Informações apreendidas e discussão}

\section{$\mathrm{O}$ idoso: usuário comum na busca pelo direito à prioridade}

Deficiências relacionadas a estrutura e organização do serviço de saúde comprometem o atendimento das necessidades dos idosos, conforme descrito no discurso a seguir.

As necessidades desse usuário não são sanadas devido a vários fatores: os maiores deles são a priorização desta clientela da fila de espera, medicação específica, uma rede de serviço especializado, transporte adequado para seu deslocamento, visitas domiciliárias em quantidade suficiente para cada equipe (ESF5, 2009).

Observamos a importância do investimento em estudos que investiguem o que é necessário e pode ser feito para o idoso no que diz respeito a recursos 
financeiros e às prioridades epidemiológicas, sociais e políticas, uma vez que esta

população é numerosa e tensionadora política - ou seja, a velhice vem assumindo novos significados diante do aumento da longevidade, tendo maior impacto sobre o Estado e sobre a vida da sociedade e dos indivíduos. Destacamos uma discussão em torno da realidade existente, uma vez que não há como lidar com todas as dificuldades e obter as respostas necessárias apenas lendo normas legais e seus comentadores. É preciso conhecer a reserva do possível, ou seja, deve haver uma avaliação crítica sobre o que é necessário e que pode ser resolvido, uma vez que, ainda que a questão esteja entre Estado e demandante, as implicações das decisões envolvem direitos sociais e afetarão diretamente o coletivo (WANG, 2009, p. 308).

Além do aspecto relacionado à saúde, esse usuário possui uma demanda social, familiar, financeira, judicial, de moradia, de transporte, enfim, multissetorial. Para atender a essas necessidades, é indispensável uma abordagem intersetorial, como percebemos na fala a seguir.

Todos são atendidos, mas a resolubilidade ainda é baixa e nem todas as necessidades dos idosos são somente em relação às doenças. Nem sempre as necessidades são sanadas, pois temos as questôes do benefício, familiares e sociais que não conseguimos resolver sozinhos (ESF5, SM1, SM2, 2009).

Em estudo realizado no Rio de Janeiro, a intersetorialidade é mencionada como sinergia necessária para o desenvolvimento social. Significa articular experiência no planejamento, realização e avaliação de ações, para alcançar resultados integrados em situações complexas. Como mostrou este trabalho, tal ideal está longe de ser alcançado no campo das políticas voltadas à população idosa, em virtude da dependência de dispositivos constitucionais do Poder Executivo que, no Brasil, são abrangentes e inseridos em uma tradição centralizadora (FERREIRA, 2003).

\section{A atenção domiciliária às pessoas idosas}

Entendemos por "atenção domiciliária a idosos" um conjunto de ações realizadas por uma equipe interdisciplinar no domicílio do usuário e família, com base no diagnóstico da realidade em que está inserido, de seus potenciais e limitaçôes. Articulam-se promoção, prevenção, diagnóstico, tratamento e reabilitação, favorecendo, assim, o desenvolvimento e adaptação de suas funções, de maneira a restabelecer sua independência e preservar sua autonomia dentro das condições físicas e ambientais possíveis (BRASIL, 2007). 
Os participantes da RA ESF consideram a realização da visita domiciliária ao idoso como um problema:

As visitas domiciliárias aos idosos acamados são um problema devido às equipes in-
completas, carência de transporte adequado e de médicos geriatras, levando a um
atendimento mais demorado. Não é possível fazer um curativo adequado por que não
tem material necessário, levando a piora do quadro do paciente (ESF1, ESF2, 2009).

Este relato revela uma infraestrutura deficitária nas Unidades Básicas de Saúde, comprometendo a qualidade da visita domiciliária, e especifica estas deficiências como de ordem material e recursos humanos. Estudos confirmam que a realidade da infraestrutura brasileira necessária para responder às demandas desse grupo etário, quanto a instalações, programas específicos e recursos humanos adequados, quantitativa e qualitativamente, ainda é classificada como precária (LOURENÇO et al., 2005, p. 311).

Evidenciamos também a escassez de profissionais especializados no cuidado com o idoso na realização da visita domiciliária, uma vez que essa clientela necessita de uma atenção direcionada da equipe, por sua fragilidade e pelo fato de na maioria das vezes estarem acamados, o que dificulta o acesso e a locomoção até o serviço de saúde mais próximo. Observamos, diante destas carências, a necessidade de adaptação e invenções criativas, sobretudo no domicílio, onde é quase um imperativo o cuidado continuado de idosos doentes e/ou fragilizados ser executado pela família. Embora não exista no país um estudo para avaliação epidemiológica da fragilidade, estima-se que $20 \%$ dos idosos apresentam algum problema de saúde que requer cuidados especializados (GONÇALVES; ALVAREZ, 2004; VERAS et al, 2007).

$\mathrm{Na}$ Suécia, assim como no Brasil, as famílias ainda são os principais prestadores de cuidados aos idosos no domicílio. A diminuição na prestação de cuidados institucionais e os cortes nos serviços públicos tiveram repercussões negativas para o cuidador familiar e podem explicar por que cuidar da família está cada vez mais comum. O desenvolvimento de serviços e apoio para os cuidadores em nível municipal tem sido estimulado através da utilização de subvenções nacionais, mas os recursos públicos têm tido dificuldade para abordar os cuidadores familiares de forma acessível, flexível e personalizada. Em 2009, o Parlamento sueco aprovou uma nova lei que obriga os municípios a oferecerem apoio às pessoas que cuidam de familiares com doenças crônicas, 
idosos ou com deficiências funcionais, representando uma mudança de paradigma de um sistema de bem-estar focado no indivíduo a um sistema mais voltado para a família (JOHANSSON et al., 2011, p. 335).

A Estratégia Saúde da Família (ESF) no Brasil tem o cadastro de saúde da população e vinculação de famílias à equipe local de referência como umas de suas diretrizes de implantação (ANDRADE et al., 2006, p. 783). Apesar disso, questões que envolvem território de atuação das equipes de ESF foram mencionadas como contribuintes para a dificuldade de atendimento do usuário pelo profissional especialista, quando este não pertence à área de abrangência, como no relato a seguir.

Em relação à visita domiciliária também há uma dificuldade porque eles não querem deixar de atender um paciente de sua área (especialistas) pra atender em uma área desconhecida (ESF1, ESF5, 2009).

Na RA SM percebemos que, nas visitas domiciliárias, o idoso perde a prioridade quando ele compartilha de um universo de prioridade:

Nós temos uma série de dificuldades que a gente vai driblando, mas, a demanda é muito grande. Principalmente, na visita domiciliária, nós temos pacientes de 90 anos, 95 anos, e fica complicado priorizar, como é que você vai priorizar de um universo que tudo é prioridade? (SM1, 2009).

Em uma população em que tudo é prioridade, deixa de ser prioridade, e sim universo. Nessas situações, devem ser considerados o pensamento crítico do serviço, as avaliações clínicas e outros indicadores de prioridade dentro deste universo, como idade mais avançada, fragilidade, nível de dependência, dentre outros. Com isso, destacamos na visita domiciliária a necessidade de hierarquizar as prioridades. O Ministério da Saúde disponibiliza instrumentos capazes de orientar a ESF na hierarquização das prioridades e planejamento desta atividade como, por exemplo, o Apgar da Família, que avalia funcionalidade familiar e orienta sobre a necessidade e frequência das visitas (BRASIL, 2006c). A utilização de instrumentos como estes contribui para a melhoria dos serviços ofertados pelo SUS à população brasileira e corroboram o planejamento de ações desenvolvidas no domicílio.

Em novembro de 2011, foi criado o programa de atenção domiciliária Melhor em Casa, que consiste em um atendimento realizado no domicílio por uma equipe multidisciplinar (EMAD) composta por médicos, enfermeiros, 
técnicos de enfermagem e fisioterapeuta. São contemplados usuários portadores de doenças crônicas, em cuidados pós-operatórios, acamados e que tenham dificuldade de locomoção para o serviço de saúde mais próximo. Contam ainda com uma equipe de apoio (EMAP) composta por fonoaudiólogo, nutricionista, odontólogo, psicólogo e farmacêutico. O programa amplia as possibilidades de atenção domiciliária à população idosa e seus cuidadores (BRASIL, 2006e).

\section{O caminho percorrido pelo idoso nas redes de atenção à saúde}

A continuidade do cuidado, também mencionada na literatura como longitudinalidade, é o acompanhamento do usuário ao longo do tempo por profissionais da equipe de atenção primária em saúde, ou demais Redes de Atenção. Está relacionada a resultados positivos, o que justifica sua utilização para fins de avaliação da Atenção Primária à Saúde. Estudos reforçam a importância do acesso aos cuidados de saúde e desenvolvimento de intervençôes que garantam a continuidade do cuidado, reduzindo os custos (CUNHA; GIOVANELLA, 2011, p. 1029; PARTHASARATHY et al., 2012, p.540).

$\mathrm{Na}$ RA ESF, observamos que o usuário não consegue o atendimento na unidade de destino em tempo hábil, e fica um longo tempo na fila de espera do sistema de marcação de consultas. Quando atendido, nem sempre esta informação e o próprio usuário retornam à Unidade Básica de Saúde para acompanhamento na comunidade. Em algumas situações, quando ele está inserido em um programa específico, o retorno das informações e o acompanhamento do usuário acontecem.

Todos os que necessitam são encaminhados, mas nem todos atendidos, porque a gente compreende que há necessidade de um atendimento rápido e que haja a contrarreferência e não ocorre, ficando o paciente perdido na fila de espera. A não ser que seja um idoso que esteja inserido em um programa de interesse, como o da Tuberculose, em casos de abandono de tratamento, imediatamente aparece no sistema, então é feita a busca ativa, a referência e a contrarreferência (ESF1, ESF2, ESF3, ESF4, ESF5, ESF6, 2009).

O idoso é encaminhado à outra rede de atenção quando a Unidade Básica de Saúde não oferece o serviço de que ele necessita, ou seja, um atendimento mais especializado e de tecnologias mais duras, sendo comum o referenciamento para os profissionais geriatras, que são raros tanto no sistema público como na assistência complementar. Esta carência gera uma imensa fila de espera de pessoas idosas aguardando atendimento especializado, ocasionando o rompimento da linha de cuidado neste percurso. 
Apesar da garantia por lei da prioridade nas filas, os idosos aguardam longos períodos por um atendimento, submetendo-se ao risco de desenvolver a síndrome da fragilidade. $\mathrm{O}$ atendimento a este indivíduo deve ser ágil e preciso, pois o próprio envelhecimento e suas comorbidades aceleram o surgimento de complicações e, consequentemente, as incapacidades, comprometendo a qualidade de vida, inclusive chegando a óbito.

$\mathrm{O}$ acesso aos serviços de saúde funciona como indicador da qualidade e resolubilidade, determinante de relevância para o cumprimento do tratamento indicado, e a falta de acesso aos níveis mais complexos de atendimento uma causa frequente de retorno de pacientes aos serviços de saúde com problemas recorrentes (ARRAIS et al., 2005, p. 1736).

A fila de espera pode ter até três mil pessoas cadastradas. Tudo para o idoso tem que ser pra ontem porque a gente não sabe se no futuro ele vai existir (ESF2, ESF4, ESF6, 2009).

A demanda de pessoas idosas só aumenta nos serviços de saúde, evidenciando a escassez de profissionais devidamente qualificados para atender às necessidades desta população. Existem poucos profissionais especializados nas áreas de Geriatria e Gerontologia, bem como a temática ainda é pouco explorada na formação dos profissionais generalistas dentro das universidades. Esta carência de profissionais de saúde habilitados para tratar idosos influencia decisivamente nas dificuldades de abordagem adequada desse paciente na RA ESP (Lourenço et al., 2005, p.311).

Nós só temos dois médicos que fazem o atendimento do município e o Centro de Referência do Idoso que tem um geriatra, mas que só atende os Idosos que são acompanhados dentro do centro de referência, ou seja, não se estende pro município (ESF3, ESF4, ESF5, ESF6, 2009).

Uma forma de equacionar a privação de profissionais especialistas, já que este é um problema de solução em longo prazo, é capacitar os profissionais da atenção primária à saúde para atender aos riscos à saúde de maior magnitude no idoso. Para tanto, as políticas devem estabelecer critérios bem determinados, claros e factíveis de atenção ao idoso no sistema público de saúde.

A RA ESP recebe usuários das outras redes assistenciais, porém, se necessitarem de uma nova demanda, retornam à Unidade Básica de Saúde, para serem redirecionados ao serviço adequado. No caso das instituições de reabilitação, o 
encaminhamento pode ocorrer para a realização de um procedimento hospitalar específico, e mantém-se o vínculo com a instituição de origem para continuidade no processo de reabilitação. Observamos, neste caso, o atendimento em três níveis de atenção, a RA ESF, RA ESP e RA HOSP, de forma contínua, sem o rompimento da linha de cuidado. $\mathrm{O}$ vínculo manteve-se fortalecido, garantindo a continuidade, individualmente.

O paciente deve retornar ao nível primário para de lá ser encaminhado para uma unidade a qual ele precise ser encaminhado. Às vezes a gente encaminha para o hospital, para fazer uma cirurgia, existindo a troca de informaçôes, depois ele volta pra fazer a reabilitação (ESP1, ESP2, 2009).

Na RA HOSP, os usuários são direcionados às outras redes de atenção conforme a necessidade de cada um, não há uma continuidade, pelas características próprias da rede. $\mathrm{O}$ usuário entra para um atendimento pontual e não tem acompanhamento longitudinal, salvo casos bem específicos. Quando a instituição não oferece o serviço de que o idoso necessita, apenas encaminha para outro sem uma devolutiva de informações.

A gente não tem contato com outro hospital, este tipo de informação de lá pra cá não existe. Eles são encaminhados, mas quando eles vão conseguir o atendimento, não sabemos (HOSP1, HOSP2, 2009).

Consideramos que os serviços gerontológicos egeriátricos ainda estão incipientes em sua instalação em nosso meio. Suas atividades necessitam de sistematização, como também de tecnologias apropriadas de cuidado. Muito ainda está por ser criado ou recriado a fim de que tais serviços, programas e instituições efetivamente funcionem como uma rede de referência e contrarreferência, permitindo que o atendimento de um usuário idoso em um dado serviço desencadeie um fluxo ágil de atenção continuada (GONÇALVES; ALVAREZ, 2004, p. 57).

Caracterizamos o atendimento móvel na RA UE como de remoção do usuário para um hospital da própria rede, ou pronto-atendimento. Ao chegar ao hospital, são avaliados e encaminhados em meio intra-hospitalar conforme a gravidade do quadro clínico.

O serviço oferecido, de forma geral, consiste na remoção. Quando chegam ao hospital, o médico faz a admissão do paciente, e os que precisam ficar internados ou vão para uma lista de espera dependendo da gravidade (UE1, UE2, 2009).

Quando é necessário um direcionamento para outras redes de atenção, não acontece o retorno de informações, rompendo-se o vínculo com o usuário. 
Não existe retorno, a gente referencia o paciente, aí eles ficam com ele lá, não

temos mais contato. A referência é de acordo com a necessidade que ele apresentar (UE1, UE2, 2009).

Na RA SM, observamos que nos Centros de Atenção Psicossociais (CAPS) Gerais a clientela idosa procura o serviço principalmente por transtornos de humor, sendo ansiedade e depressão os mais prevalentes, com presença marcante no sexo feminino. Nos CAPS Álcool e Drogas (CAPS AD), destacamos o tratamento do alcoolismo e tabagismo. Os participantes da pesquisa revelam que os mais jovens são atendidos pela dependência de drogas ilícitas, enquanto os idosos demandam atenção em relação às drogas permitidas, mas tão deletérias quanto as não permitidas.

A clientela maior assim, de pessoas idosas, seria no grupo de tabagismo e de alcoolismo, as drogas ilícitas seria mais assim do jovem, do adulto jovem. O transtorno mais comum dessa população, do CAPS geral, é depressão e transtorno bipolar, são pessoas idosas e a maioria mulheres (SM1, SM3, SM4, SM5, 2009).

A RA SM realiza, além do acompanhamento multiprofissional, terapia individual e grupal, a visita domiciliária e o atendimento psicossocial, por meio do matriciamento nas Unidades Básicas de Saúde. Nos casos de transtornos leves, são realizados trabalhos de escuta, resgate da autoestima, geração de renda e lazer.

E a rede hospitalar é uma rede que também não tem vínculo nem com a atenção básica, nem com a saúde mental, hoje a gente tem o apoio matricial, a saúde mental e a atenção básica, que fez nos aproximarmos mais, e dialogarmos. Mas nós não temos diálogo, por exemplo, com a internação de um idoso que está com síndrome de abstinência alcoólica (SM4, SM5, 2009).

A RA ESF e a RA SM, com o matriciamento, estreitaram seus vínculos, efetivando não só o encaminhamento e o acompanhamento dos usuários, mas também as relações intersetoriais, e estão trabalhando em conjunto num modelo usuário-centrado, estabelecendo vínculo, acolhimento e responsabilização.

Com o matriciamento, estreitaram mais esses vínculos. Então hoje é muito mais fácil fazer o referenciamento pra Unidade Básica de Saúde quando precisa de exames. Então, quando a gente precisa encaminhar, o CAPS liga pra Unidade, e a Unidade entra em contato com esse profissional de referência, que cuida da saúde do idoso do território (SM1, SM3, SM4, SM5, 2009).

Observamos que as RA ESF e a RA SM articulam-se de forma a realizar arranjos com perspectivas promissoras à população idosa, beneficiandose de um adequado atendimento de saúde ao longo de seu processo de vida. 
Desafios imensos e inumeráveis apresentam-se na área da operacionalização das normatizações, as quais dependem, entre muitos fatores, de recursos institucionais físico-financeiros, de recursos humanos capacitados e gestão continuada de serviços implantados (GONÇALVES; ALVAREZ, 2004, p. 57).

\section{Considerações finais: o elo a ser construído}

Os participantes deste estudo compreendem a necessidade de uma abordagem intersetorial e multiprofissional no cuidado destinado ao idoso, passo importante para a implementação de políticas destinadas a esta população no município. Além disso, as necessidades dos idosos, no setor saúde, não são contempladas apenas em uma rede de atenção, devendo ser complementadas e continuadas nas outras redes, percurso denominado "linha de cuidado", que torna possível uma abordagem integral ao indivíduo.

Percebemos a visita domiciliária na RA ESF e na RA SM como um problema, por diversos fatores: equipe multidisciplinar incompleta, dificuldade em selecionar as famílias/idosos que realmente necessitam deste cuidado e infraestrutura deficitária desses serviços.

$\mathrm{Na}$ França, estudo sobre redes perinatais integradas destacou a continuidade, os serviços de integração e a colaboração interprofissional como elementos essenciais, inseparáveis e inter-relacionados nas transformações que ocorrem no sistema de saúde francês. Ressaltou ainda a eficiência do funcionamento de um sistema de saúde organizado por meio de redes e a necessidade de direcionamento de recursos para garantir sua operacionalização ideal em curto e longo prazos. A mesma pesquisa revelou que as dificuldades de implementação de um sistema de gerenciamento de continuidade do cuidado estão mais relacionadas aos valores profissionais - ou seja, falta consenso entre profissionais de diferentes disciplinas sobre a participação ativa dos usuários, a responsabilidade no uso de ferramentas específicas de informação, e compartilhamento na identificação e apoio à pacientes em risco.

Sugerimos ainda o desenvolvimento de conhecimentos e estratégias acerca do reconhecimento do papel dos pacientes na continuidade dos cuidados, promoção da integração dos médicos na prática privada para atendimento em rede integrada e aumentar a colaboração interprofissional (GOULET et al., 2006).

Sobre a continuidade do cuidado, destacamos que ela acontece, mas de forma ainda tímida e com falhas. $\mathrm{O}$ idoso consegue percorrer as cinco redes de 
atenção à saúde do sistema municipal de saúde de Fortaleza, mas de forma lenta,

enfrentando filas de espera neste percurso. Vemos ainda a dificuldade do retorno do usuário e de suas informações para a Rede de Atenção de origem. O acesso existe e é livre, mas o sistema de saúde ainda não conseguiu se organizar para que a continuidade do cuidado aconteça de forma contínua.

A produção da saúde, na perspectiva da integralidade da atenção, envolve formatos institucionais que articulam respostas macro e microinstitucionais, ou seja, para equacionar o caminhar na linha de cuidado, considera-se tanto o processo de trabalho em saúde como as demandas de organização do sistema de saúde e suas interfaces. A linha de cuidado se completa na medida em que os macroprocessos se articulam com microprocessos, mas sempre se corre o risco da sua ruptura ou fragmentação, pois são muitas variáveis a serem garantidas e nem sempre elas se entrelaçam (MALTA; MERHY, 2010, p. 593).

Para o fortalecimento desta malha de cuidados do idoso, é necessário um estreitamento das relações entre as redes, por meio, principalmente, do repasse de informações que subsidiem o acompanhamento do idoso na comunidade, favorecendo uma atenção integral. Enfatizamos que o repasse das informações está diretamente relacionado ao envolvimento e integração entre os profissionais de saúde que constroem o sistema público de saúde brasileiro. ${ }^{2}$

\section{Referências}

ANDRADE, L. O. M. Modelo de gestão e de atenção integral à saúde. In: FORTALEZA. Plano Municipal de Saúde 2007. Fortaleza: Secretaria Municipal de Saúde, 2008. p. 46-48. ANDRADE, L. O. M.; BARRETO, I. C. H. C.; BEZERRA, R. C. Atenção primária à saúde e estratégia saúde da família. In: CAMPOS, G.W.S. et al. (Org.). Tratado de Saúde Coletiva. São Paulo: Hucitec, 2006. p. 783-836.

ARRAIS, P. S. D. et al. Prevalência e fatores determinantes do consumo de medicamentos no Município de Fortaleza, Ceará, Brasil. Cad. Saúde Pública, v. 21, p. 1736-1746, 2005.

BRAGA, E. C. Critérios de suficiência para análise de redes assistenciais. Consulta Pública no 26. Exposição de Motivos. Agência Nacional de Saúde Suplementar. Rio de Janeiro: ANS, 2006.

INSTITUTO BRASILEIRO DE GEOGRAFIA E ESTATÍSTICA. Sala de Imprensa: Tábuas completas de Mortalidade 2009. Brasília: IBGE, 2009. Disponível em: <http:// www.ibge.gov.br>. Acesso em 15 fev. 2012. 
BRASIL. Lei no 8.842, de 04 de janeiro de 1994. Dispõe sobre a política nacional do idoso, cria o Conselho Nacional do Idoso e dá outras providências. Brasília: 1994.

_. Ministério da Saúde. Envelhecimento e saúde da pessoa idosa. Brasília: MS, 2006c.

_. Ministério da Saúde. Estatuto do idoso. Brasília: MS, 2003.

. Ministério da Saúde. Portaria no 2.529, de 19 de outubro de 2006. Institui a internação domiciliar no âmbito do SUS. Brasilia: MS, 2007.

. Ministério da Saúde. Portaria no 2.528, de 19 de outubro de 2006. Aprova a Política Nacional de Saúde da Pessoa Idosa. Brasília: MS, 2006a.

. Ministério da Saúde. Portaria no 399, de 22 de fevereiro de 2006. Divulga o Pacto pela Saúde 2006 - Consolidação do SUS e aprova as Diretrizes Operacionais do Referido Pacto. Brasília: MS, 2006b.

- Ministério da Saúde. Secretaria de Atenção à Saúde. Departamento de Atenção Básica. Prograb: Programação de gestão por resultados. Brasília: MS, 2006 d.

- Ministério da Saúde. Secretaria de Atenção à Saúde. Departamento de Atenção Básica. Programa Melhor em Casa. Brasília: MS, 2006e.

. Secretaria de Gestão Estratégica e Participativa. Decreto no 7.508, de 28 de junho de 2001. Regulamentação da Lei 8.080/90. Brasília: MS, 2011.

CAMARANO, A. A.; PASINATO, M. T. O envelhecimento populacional na agenda das políticas públicas. In:

(Org.). Os novos idosos brasileiros: muito além dos 60? Rio de Janeiro: Ipea, 2004. p. 253-292.

CHRISTOPHER, M. C.; MICHAEL, W.; STEVEN, R. C. Defining the domain of geriatric medicine in an urban public health system affiliated with an academic medical center. Special Series: the association of directors of geriatric academic programs symposium. Domain of Geriatric Medicine, v. 56, n. 10, p. 1802-1806, 2008.

CUNHA, E. M.; GIOVANELLA, L. Longitudinalidade/continuidade do cuidado: identificando dimensões e variáveis para a avaliação da Atenção Primária no contexto do sistema público de saúde brasileiro. Rev Ciência e Saúde Coletiva, v. 16, n. 1, p. 1029-1042, 2011.

FERREIRA, M. L. C. Intersetorialidade: um desafio na implementação da política nacional do idoso no estado do Rio de Janeiro. Dissertação (Mestrado) - Escola Nacional de Saúde Pública, Fundação Oswaldo Cruz, Rio de Janeiro, 2003.

FORTALEZA. Prefeitura Municipal de Fortaleza. Secretaria Municipal de Saúde de Fortaleza. Relatório de gestão 2006:"saúde, qualidade de vida e a ética do cuidado". Fortaleza: SMS, 2007.

GONÇALVES, L. H. T.; ALVAREZ, A. M. A Enfermagem gerontogeriátrica: perspectiva e desafios. Rev. Bras. Ciênc. Env. Hum., v. 1, n. 1, p. 57-68, 2004. 
GOULET, L. et al. Évaluation d'unsystème de gestion de la continuité des soins. Groupe de recherche interdisciplimaire em santé. Secteur Santé Publique, Faculté de médecine, Université de Montréal, Montréal. 2006.

JOHANSSON, L.; LONG, H.; PARKER, M. G. Informal care giving for elders in Sweden: an analysis of current policy developments. J Aging Soc Policy, v. 23, n. 4, p. 335-353, 2011. LEFEVRE, F.; LEFEVRE, A. M. C. O discurso do sujeito coletivo: um novo enfoque em pesquisa qualitativa (desdobramentos). Caxias do Sul: EDUSC, 2003.

LOURENÇO, R. A. et al. Assistência ambulatorial geriátrica: hierarquização da demanda. Rev. Saúde Pública, v. 39, n. 2, p. 311-318, 2005.

MALTA, D. C.; MERHY, E. E.The path of the line of care from the perspective of nontransmissible chronic diseases. Interface-Comunic., Saude, Educ., v. 14, n. 34, p. 593-605, 2010.

PARTHASARATHY, S. et al. The role of continuing care in 9-year cost trajectories of patients with intakes into an outpatient alcohol and drug treatment program. Med Care, v. 50, n. 6, p. 540-546, 2012.

SANTOS, C. T. B. A Linha de Cuidado do Idoso nas Redes Assistenciais de Fortaleza: visão dos gestores. Dissertação (Mestrado) - Faculdade de Medicina, Departamento de Saúde Comunitária, Universidade Federal do Ceará, Fortaleza, 2009.

VERAS, R. P. et al. Promovendo a Saúde e Prevenindo a Dependência: identificando indicadores de fragilidade em idosos independentes. Rev. Bras. Geriatr. Gerontol, v. 10, n. 3, p. 355-370, 2007.

WANG, D. W. L. Reserva do possível. Mínimo existencial e direito à saúde: algumas aproximaçôes. Revista de Direito Sanitário, v. 10, n. 1, p. 308-318, 2009.

WORLD HEALTH ORGANIZATION. Global Health Observatory (GHO). Life expectancy at birth: 1990, 2000, 2009. Disponível em: <http://www.who.int>. Acesso em: 11 dez. 2012.

\section{Notas}

${ }^{1}$ Este artigo científico corresponde aos resultados parciais da dissertação de mestrado $A$ linha de cuidado do idoso nas redes assistenciais de Fortaleza-CE: visão dos gestores, aprovada pelo Comitê de Ética em Pesquisa da UFC/COMEPE Protocolo 45/09, financiada inicialmente pela Capes e em seguida pela Secretaria Municipal de Saúde de Fortaleza.

${ }^{2}$ C. T. B. dos Santos participou da concepção e execução das etapas do projeto, análise e interpretação dos dados, redação do artigo. L. O. M. de Andrade participou da concepção do projeto, orientação da dissertação de mestrado e redação do manuscrito, revisão crítica relevante do conteúdo intelectual e aprovação final da versão a ser publicada. M. J. da Silva co-orientou a redação do artigo, realizou revisão crítica relevante do conteúdo intelectual e aprovação final da versão a ser publicada. M. F. de Sousa participou da revisão crítica relevante do conteúdo intelectual e aprovação final da versão a ser publicada. 
Course of the elderly in health care network: a link to be built

We aimed to understand the continuity of care provided to the elderly in health care networks in Fortaleza-CE, Brazil. This is a descriptive qualitative study that used focus groups, semi-structured interviews and discourse of the collective subject for data collection and organization. In Family Health Care Network, we observed deficits in infrastructure and professionals. In Specialized Care, we verified repressed demand due the shortages in specialized services. In Hospital, the flow of information is deficient. In Urgency and Emergency, the mobile service prioritizes the removal and in the hospital the care according to severity. The close relationship between the Mental Health and Family Health Networks contributes to the effective continuity of care. We conclude that the care for the elderly in the network is fragile and discontinued, and impairs the consolidation of integral care.

> Key words: health services for the aged; health care delivery; elderly. 